Musées, Patrimoine et Culture scientifiques et techniques

$171 \mid 2017$

mai-juin 2017

\title{
30 ans de production d'expositions à la Cité des Sciences et de l'Industrie
}

Dominique Botbol, Marc Girard and Diane Chollet

\section{OpenEdition \\ Journals}

Electronic version

URL: http://journals.openedition.org/ocim/1770

DOI: $10.4000 /$ ocim. 1770

ISSN: 2108-646X

\section{Publisher}

OCIM

Printed version

Date of publication: 1 May 2017

Number of pages: 5-14

ISSN: 0994-1908

Electronic reference

Dominique Botbol, Marc Girard and Diane Chollet, «30 ans de production d'expositions à la Cité des Sciences et de I'Industrie », La Lettre de I'OCIM [Online], 171 | 2017, Online since 01 May 2018, connection on 05 May 2019. URL : http://journals.openedition.org/ocim/1770 ; DOI : 10.4000/ ocim. 1770

This text was automatically generated on 5 May 2019.

Tous droits réservés 


\title{
30 ans de production d'expositions à la Cité des Sciences et de l'Industrie
}

\author{
Dominique Botbol, Marc Girard and Diane Chollet
}

La Cité des Sciences et de l'Industrie aujourd'hui avec Solar Impulse.

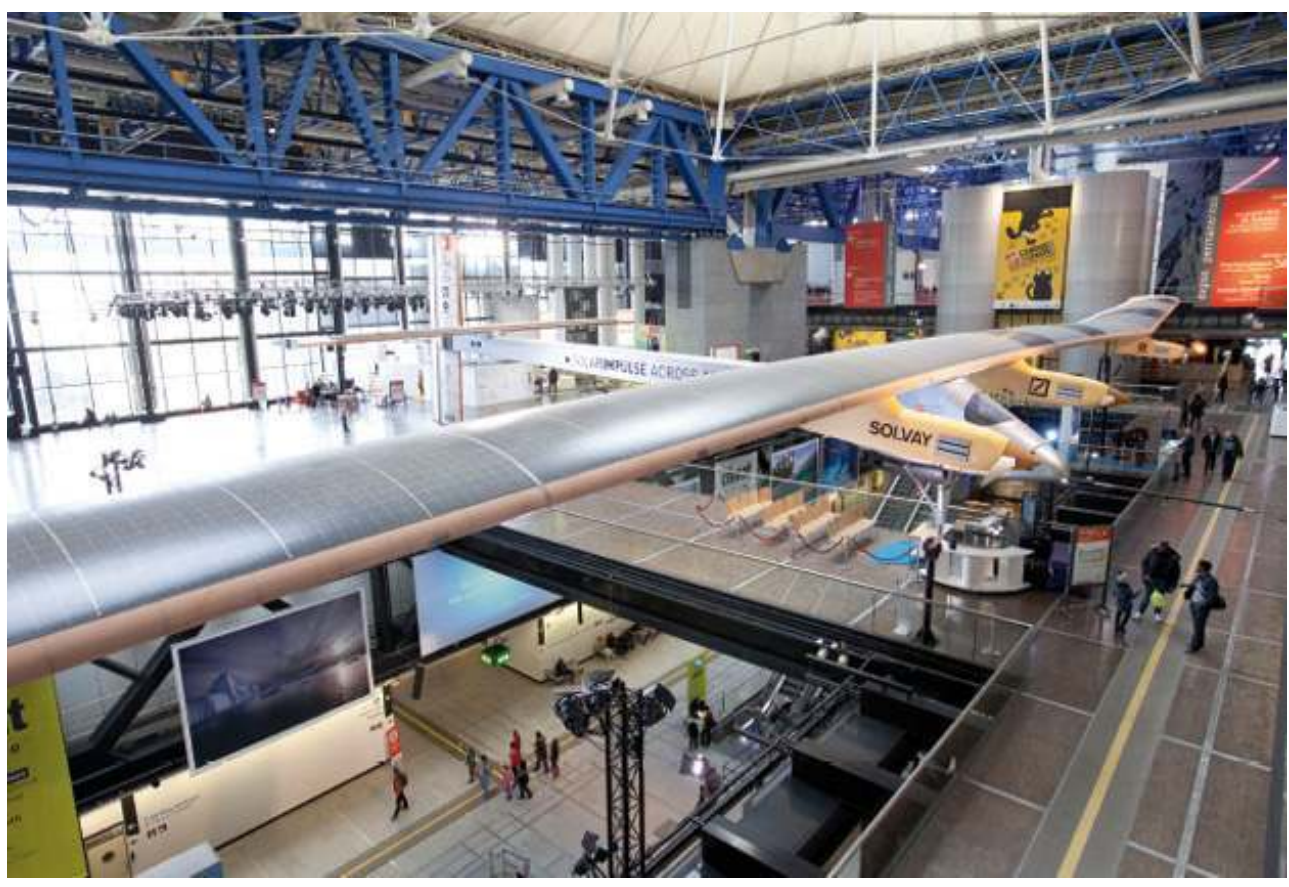

(c) EPPDCSI/Nicolas Breton

L'histoire de la Cité des Sciences et de l'Industrie s'inscrit dans l'histoire de la muséographie des sciences et plus particulièrement dans le mouvement de vulgarisation des sciences initié au XIX siècle avec les grandes expositions internationales. L'Exposition universelle de 1937 a suscité la création du Palais de la découverte. Jean Perrin avait la volonté de montrer à tous les publics "la science en train de se faire" en présentant de manière spectaculaire des expériences réelles pour susciter des vocations. 
Ouverte en 1986, la Cité des Sciences et de l'Industrie s'inscrit dans cette volonté de rendre accessible à tous la culture scientifique et technique. Mais elle s'inspire pour cela des Sciences Centers américains et canadiens, et plus particulièrement de l'Exploratorium de San Francisco créé par le physicien Frank Oppenheimer, mais aussi de l'Ontario Science Center, tous deux ouverts en 1969 et précurseurs de la muséologie "Hands On", qui refonde la relation avec le public au travers de l'interactivité.

Depuis son origine, sans collection, sans mission de conservation, la Cité n'a eu pour seule légitimité que sa mission culturelle et son public qu'elle a dû attirer, convaincre et fidéliser. Outre l'intérêt de sa programmation, elle a fondé l'attractivité de ses expositions sur l'originalité et la créativité de sa muséographie. 
Explora, l'exposition permanente en 1986.
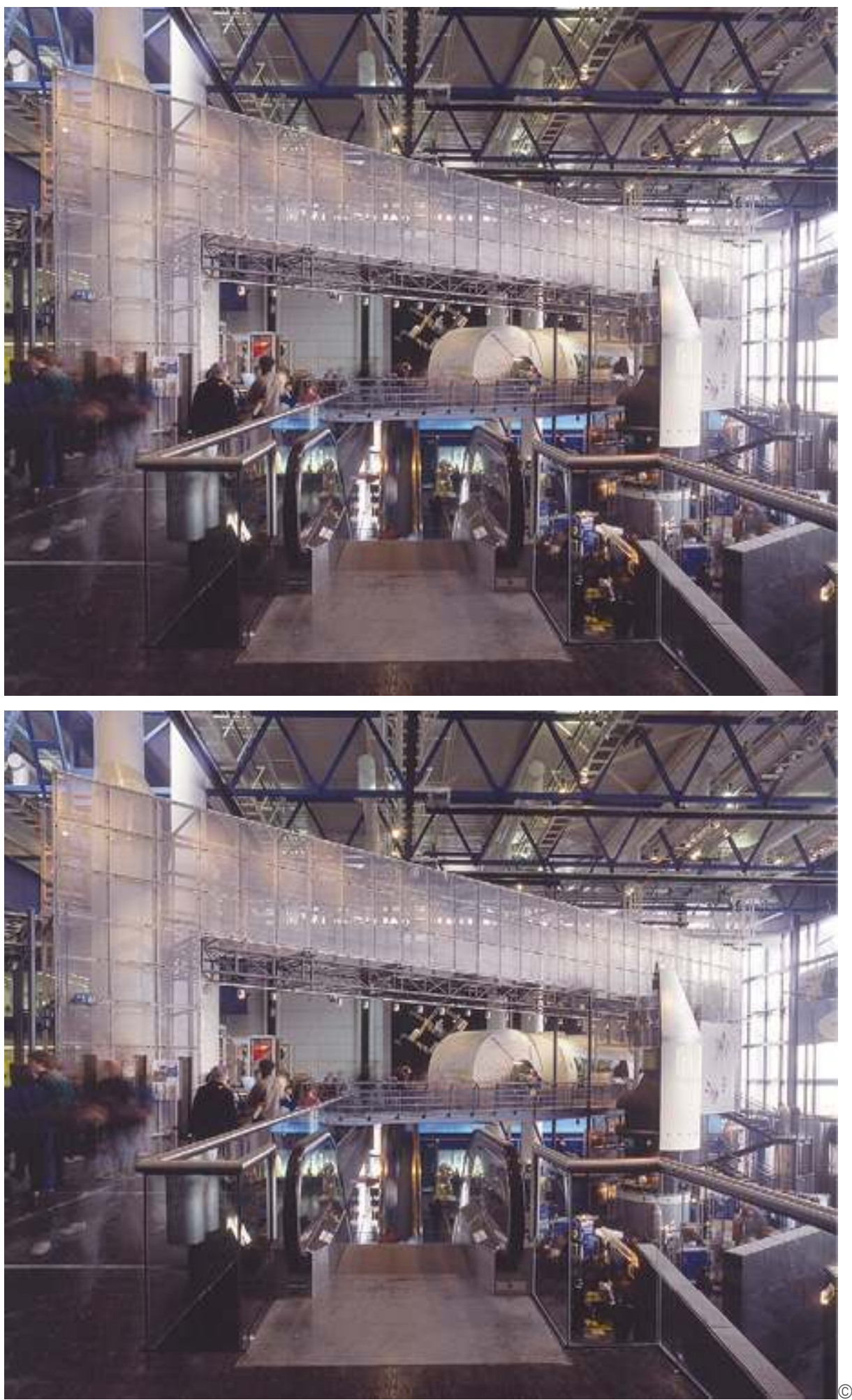

EPPDCSI

3 Le principe d'une visite autonome et interactive, alors inédit en France, a ainsi d'emblée caractérisé son identité et assuré son succès. Cette muséographie s'est toujours efforcée de se faire l'écho des évolutions du champ technoscientifique et de considérer les attentes 
des visiteurs. Ainsi, la Cité est devenue un laboratoire de création de formes muséographiques souvent interactives, avec des palettes très étendues de formats, styles, sujets, approches, adaptées à des publics très variés.

Autre élément marquant : la confrontation des propositions muséographiques à des espaces gigantesques, ménagés dans un bâtiment industriel réhabilité. Cela appelait des solutions formelles de grande échelle. La muséographie fut aussi un moyen de rendre confortable et intéressante la visite des espaces de la Cité des Sciences et de l'Industrie. On ne perdra pas de vue également que l'établissement a bénéficié au cours de ces 30 ans, malgré des variations conjoncturelles, d'un soutien financier substantiel de la puissance publique. Cette confiance des tutelles de la Cité des Sciences et de l'Industrie (ministère de la Culture et ministère de l'Éducation nationale de l'Enseignement supérieur et de la Recherche) lui a permis de maintenir, bon an, mal an, les capacités de production correspondantes à ses missions.

Il n'y a pas eu à ce jour de publication sur le sujet et l'anniversaire des 30 ans de la Cité nous donne l'occasion de faire le point sur la politique d'expositions de la Cité depuis son origine, du point de vue des muséographes praticiens que nous sommes. Ce sont ainsi plus de 300 expositions produites, mais nous ne pourrons en citer qu'une petite partie d'entre elles : que ceux qui ont contribué à cette production prolifique nous en excusent. On peut distinguer trois grandes périodes au cours de ces 30 ans.

\section{Première période : invention et défrichage (1986 à 1991)}

Les cinq premières années sont celles de l'invention et du défrichage de la production d'expositions interactives à caractère scientifique et technique.

\section{Les expositions permanentes 1 ère génération}

Développée sur les 3 niveaux d'un espace de $20000 \mathrm{~m}^{2}$ baptisé Explora, la première génération des expositions permanentes est organisée en quatre grands secteurs thématiques pluridisciplinaires avec une ambition encyclopédique, où les sciences humaines côtoient les sciences dure : transversalement au bâtiment, trois secteurs sur trois niveaux, "De la Terre à l'Univers", "L'aventure de la Vie", "Matière et travail de l'homme", et un seul secteur longitudinal sur un niveau "Langages et communication".

Il apparaît très vite que les secteurs transversaux ne sont pas perçus par les visiteurs car ils ne correspondent pas à leurs parcours naturels, par plateau horizontal. Ils sont souvent perdus. Ainsi, la Cité a ouvert en prenant à rebours ce bâtiment gigantesque qu'elle n'a depuis cessé de tenter d'apprivoiser.

9 Inspirées pour une grande part de la muséologie "Hands-On" des centres de sciences anglo-saxons, les expositions comportent un grand nombre de manips physiques interactives typiques des musées des sciences, chaque manip délivre un fragment de discours. Il n'y a pas de frontière très marquée entre chaque exposition et les maîtres mots sont liberté des parcours et interactivité.

La scénographie utilise massivement le "SIRT" (Système Interne de Réseaux Tertiaires). Ce système de construction (poteaux, poutres, panneaux de remplissages, goulottes pour 
les réseaux) a été spécifiquement conçu pour équiper les $20000 \mathrm{~m}^{2}$ d'Explora. Avec un investissement initial lourd de 150 millions de francs, il est toujours en usage 30 ans plus tard.

11 Dans l'esprit d'une scénographie monumentale, de grands et impressionnants objets sont installés sur la galerie sud comme la coiffe de la fusée Ariane ou la maquette à l'échelle 1 du Nautile.

Déjà, notre muséographie "French touch" se démarque par une attention particulière portée au design très soigné des manips, avec de magnifiques réalisations comme la Fontaine turbulente ou la Bulle de son. En outre, elle présente de tous petits écrans informatiques interactifs qui constituent une attraction car l'informatique individuelle est alors très peu répandue en France. Mais la fiabilité des éléments interactifs n'est pas satisfaisante et le taux de panne est vite trop élevé.

\section{Les expositions temporaires 1 ère période}

Parallèlement, une programmation d'expositions temporaires très active, ambitieuse et foisonnante, est mise en œuvre. Les expositions temporaires sont installées un peu partout aux niveaux inférieurs du bâtiment et peu sur Explora qui est alors principalement dédié aux expositions permanentes. $\mathrm{Au}$ rez-de-chaussée, sont programmées de grandes expositions très attractives. Les muséographes défrichent le terrain encore largement inexploré de "l'exposition culturelle à contenus scientifiques".

14 Certaines de ces expositions créent l'événement et sont restées dans les mémoires. On peut mentionner à ce titre La vigne et le vin (1988) qui proposait une approche transversale d'un thème servi par une muséographie diversifiée. Cette exposition a fait date parce que, de manière alors peu fréquente, le réel était importé dans l'exposition sous la forme d'une véritable vigne, d'un chais authentique ou d'un atelier de dégustation présenté in situ.

La Vigne et le vin

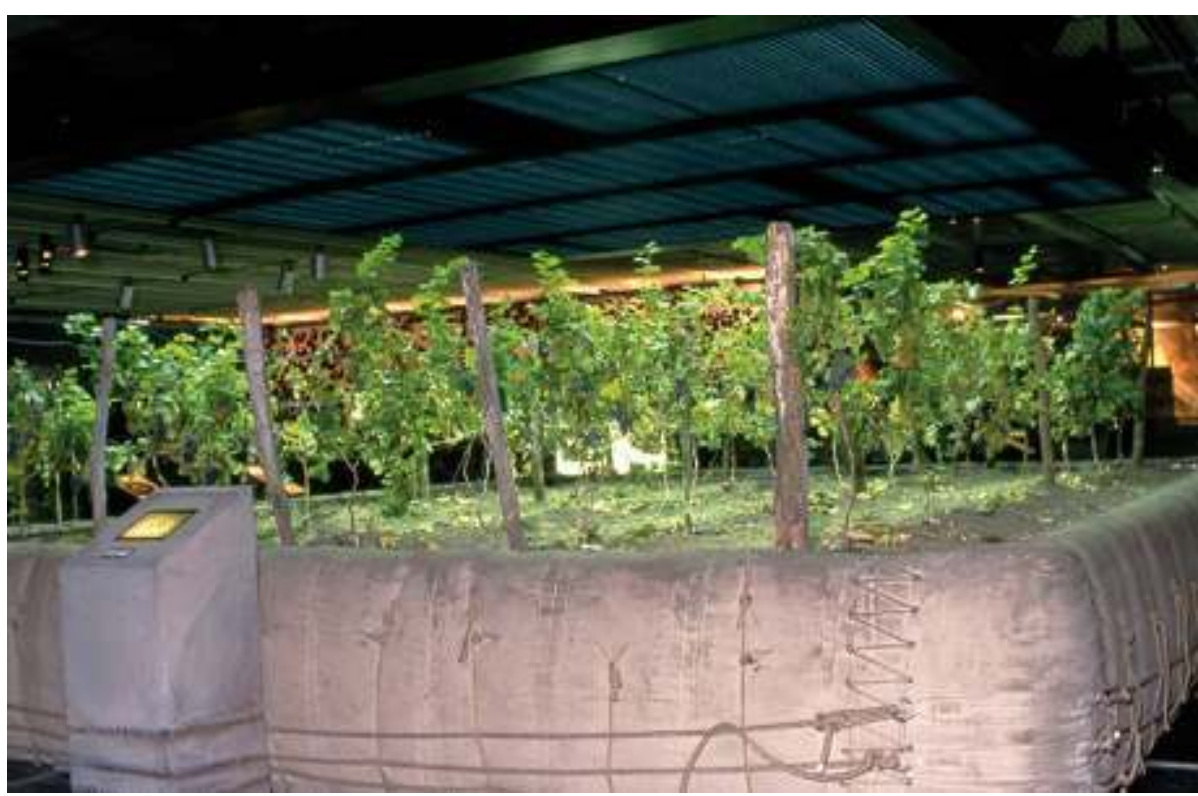

(C) EPPDCSI/ Descamps 

permanence animée par des comédiens, jouant des scénettes de reconstitutions historiques. L'exposition Vive l'eau, quant à elle, était traversée de part en part par un fleuve de deux mètres de large et de soixante-cinq mètres de long, véritable maquette/ modèle physique d'un fleuve avec ses équipements.

Les Savants et la Révolution

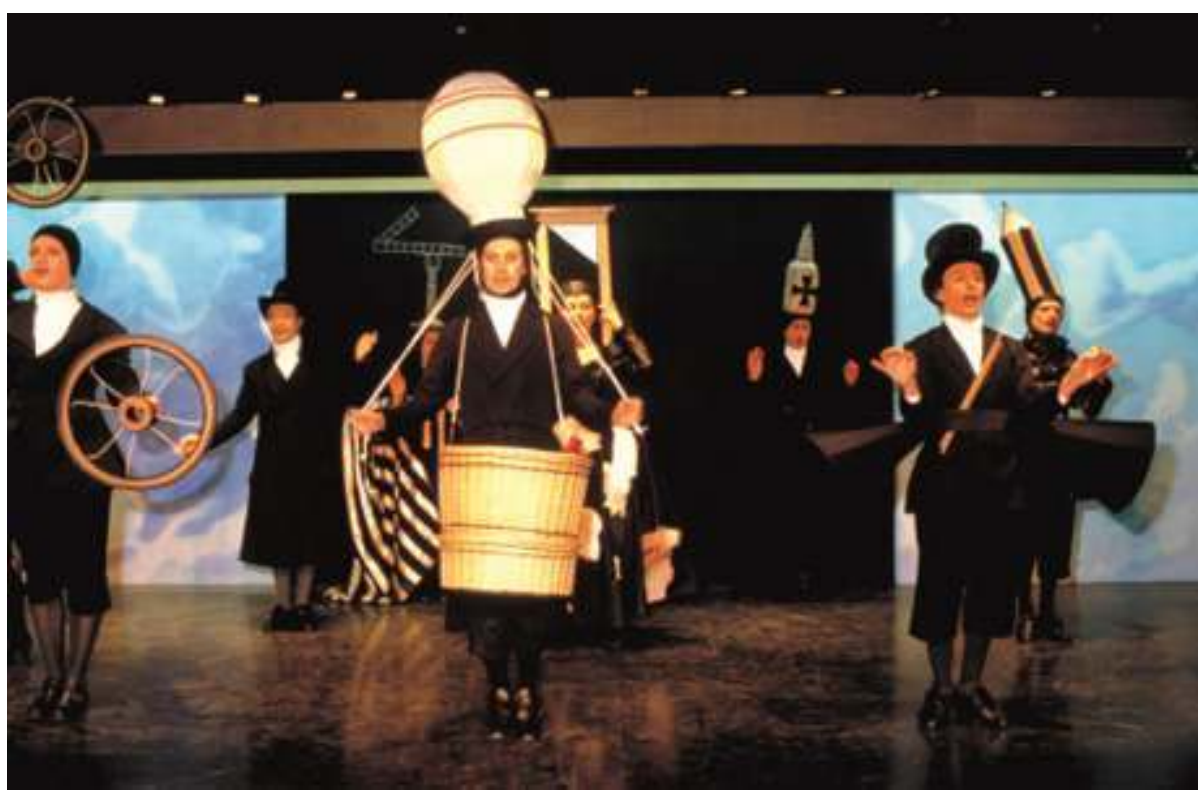

(c) EPPDCSI/Bernard Baudin

À partir de 1990, Naissance d'un bateau, sur le thème de la construction nautique, inaugure une série d'expositions où le public assistait en direct à une activité professionnelle : ici, la construction de voiles et de coques de bateau par des professionnels installés en résidence, sur Explora. L'expérience a été ensuite poursuivie avec Les Métiers du Son et Les Métiers de l'Hôpital. 


\section{Deuxième période : fiabilisation et contextualisation (1992 à 2001)}

23 Les dix années suivantes sont celles de la fiabilisation technique des éléments interactifs et de la contextualisation des contenus scientifiques, dans une approche "Science et société".

\section{Les expositions permanentes $2^{\mathrm{e}}$ génération}

La Cité apprivoise son bâtiment et connaît mieux ses publics. Elle entreprend de renouveler ses expositions permanentes en faisant évoluer la muséographie initiale.

Une organisation en trois unités de visite horizontales, correspondant à des unités de sens, exploite la déambulation naturelle des visiteurs. Le mini-guide des expositions permanentes précise "sur la galerie sud, six pôles d'activités et de réflexion de la société industrielle contemporaine. Sur la galerie nord, outils sensoriels, conceptuels et techniques qui permettent à l'homme d'appréhender le monde et le communiquer. Sur le balcon nord: l'Homme, la Terre, l'Univers".

\section{L'Homme et la santé}

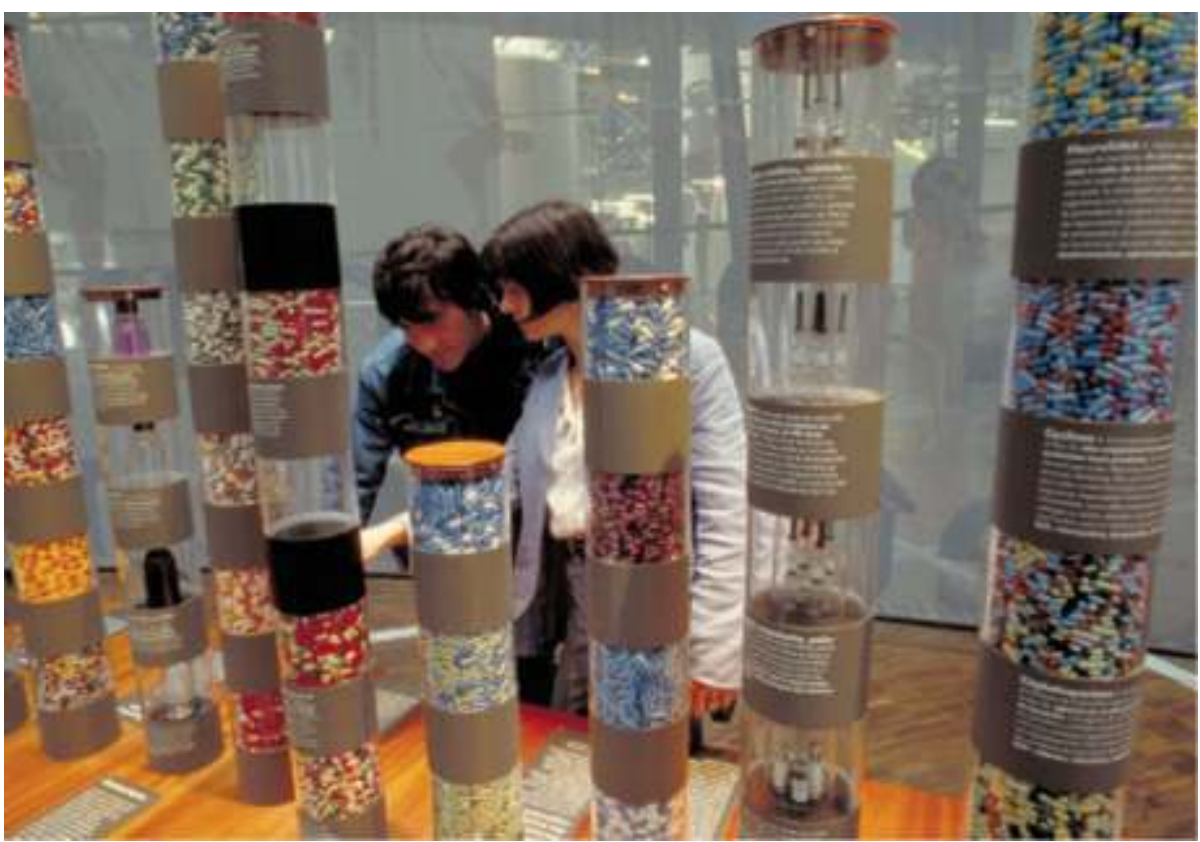

(c) EPPDCSI/Arnaud Legrain

Les contenus sont contextualisés, en prise avec la réalité de la recherche et l'industrie et les questions de société, ils s'adressent au visiteur citoyen. Une muséographie plus discursive, plus contextuelle, moins fragmentée, propose un parcours plus explicite et narratif à l'intérieur de chaque exposition tandis que la fiabilisation technique des dispositifs entraîne une baisse significative du taux de panne.

Les moyens financiers restent importants et permettent, à partir de 1992 et jusqu'en 2001, d'entreprendre une série de "renouvellements" (création d'un nouveau thème à la place 
du thème initial avec Environnement ou L'Homme et la santé) ou "d'aménagements" (reprise de la muséographie en conservant le thème initial avec une deuxième génération des expositions Les Sons et Images). La scénographie utilise toujours abondamment le système de construction interne (SIRT), mais propose des univers plus marqués, servant en cela cette volonté éditoriale d'ouverture sur le monde et sur les applications industrielles des sciences fondamentales. Ces évolutions sont accompagnées par l'installation de grands objets spectaculaires exposés à la belle lumière de la Galerie Sud, comme le Mirage IV.

Dès 1992, des grands objets spectaculaires sont exposés : le Mirage IV.

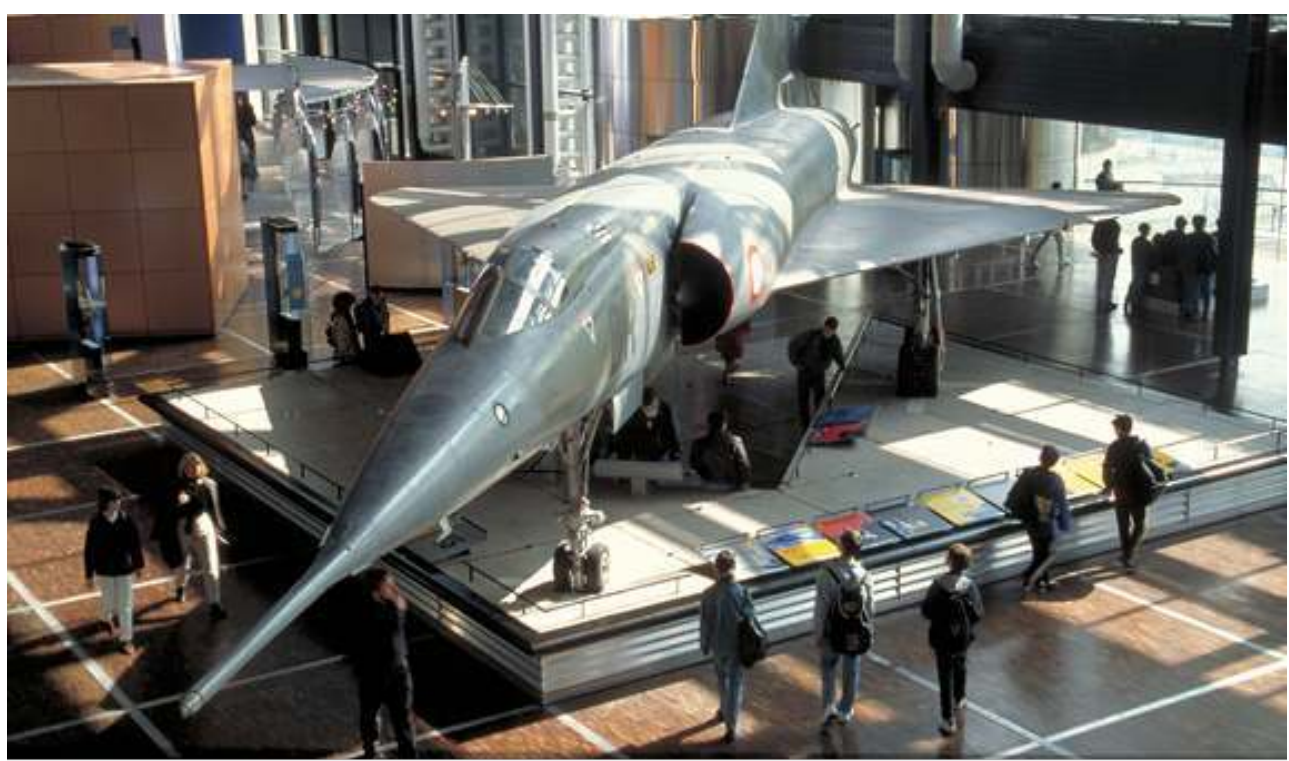

(c) EPPDCSI/Michel Lamoureux

\section{Les expositions temporaires $2^{\mathrm{e}}$ période}

Forte des enseignements de la période précédente, une politique d'expositions temporaires de "Très Grand Format" est développée à partir de 1994, en associant plusieurs galeries et parfois même en créant des planchers supplémentaires comme pour Emballe-moi! ou Mesures et démesure.

Emballe-moi ! importait dans le musée une fraction de la réalité, en jouant la sublimation esthétique du monde industriel de l'emballage : un spectaculaire mur d'écrans mettait en scène et mimait la transformation des matières premières dans les usines produisant les emballages; de vrais convoyeurs industriels transportaient bouteilles et emballages sous les pieds et les yeux des visiteurs.

En termes de contenu, l'exposition visait à présenter une industrie puissante et diversifiée sur deux galeries superposées :

31 - son histoire sur $700 \mathrm{~m}^{2}$;

32 - son actualité spectaculaire, des convoyeurs sous une dalle de verre ;

33 - un show multi-écrans répliquant les différentes filières d'emballages ;

34 - les problématiques du recyclage avec le partenariat d'Éco Emballages. 
Cette exposition dut une partie de son succès à la campagne de publicité provocante Emballe-moi ! - qui lui fût consacrée. Elle le doit aussi à sa qualité muséographique née de la collaboration harmonieuse d'une équipe externe experte et créative avec une équipe interne dont l'expérience muséographique permettait la mise en œuvre professionnelle de toutes les audaces.

Pour Mesures et démesure, la scénographie rendait d'emblée justice au titre de l'exposition, puisque nous avions dû créer de toutes pièces au dessus de la galerie principale, un troisième plateau pour faire la place nécessaire à l'ambition des contenus scientifiques. Il s'agissait réellement d'une exposition d'auteurs, de fait la combinaison - orageuse, quelquefois - d'un propos scientifique et d'un geste scénographique dû à l'architecte Jean Nouvel : la qualité de l'exposition devait beaucoup à la création de ce grand nom de l'architecture contemporaine servant le propos scientifiquement très travaillé et rigoureux.

7 En 1997, le parcours de Nouvelles Images, Nouveaux Réseaux se déployait sur deux galeries superposées : les nouvelles techniques digitales des télécommunications et du multimédia au premier niveau, les usages et enjeux, au second. Un élément marquant de cette exposition, qui témoigne des premières représentations des possibilités de l'Internet, est l'appel à un artiste, Maurice Benayoun, pour donner une représentation de ce monde virtuel vertigineux qui commençait alors à se dessiner : une salle de navigation constituée de grands écrans suggérant tous les savoirs du monde rangés dans des rubriques contenues sur des cubes visualisés en trois dimensions et manipulables par le visiteur.

8 En 1999, s'ouvre l'ambitieuse exposition Désir d'apprendre. Pour couvrir largement le champ de l'apprentissage, pas moins de quatre commissaires pour une exposition de $2000 \mathrm{~m}^{2}$ sur deux niveaux. Chaque commissaire avait en charge une partie du programme, et chaque partie avait une muséographie qui lui était propre. Deux éléments marquants du point de vue muséographique : des manips originales et collectives, scénographiées à grande échelle et le travail avec un artiste, Anton, pour reconstituer l'histoire de l'école depuis l'Antiquité.

En complément de ces grandes expositions, une programmation opportuniste d'expositions temporaires de format moyen permettait de répondre à des commandes institutionnelles, des sollicitations d'entreprises, notamment à l'occasion d'anniversaires comme Maroc, l'esprit de l'eau en 1999. Puis, entre 2000 et 2001, l'absence d'expositions temporaires de grand format entraîne à nouveau une baisse de la fréquentation d'Explora. 


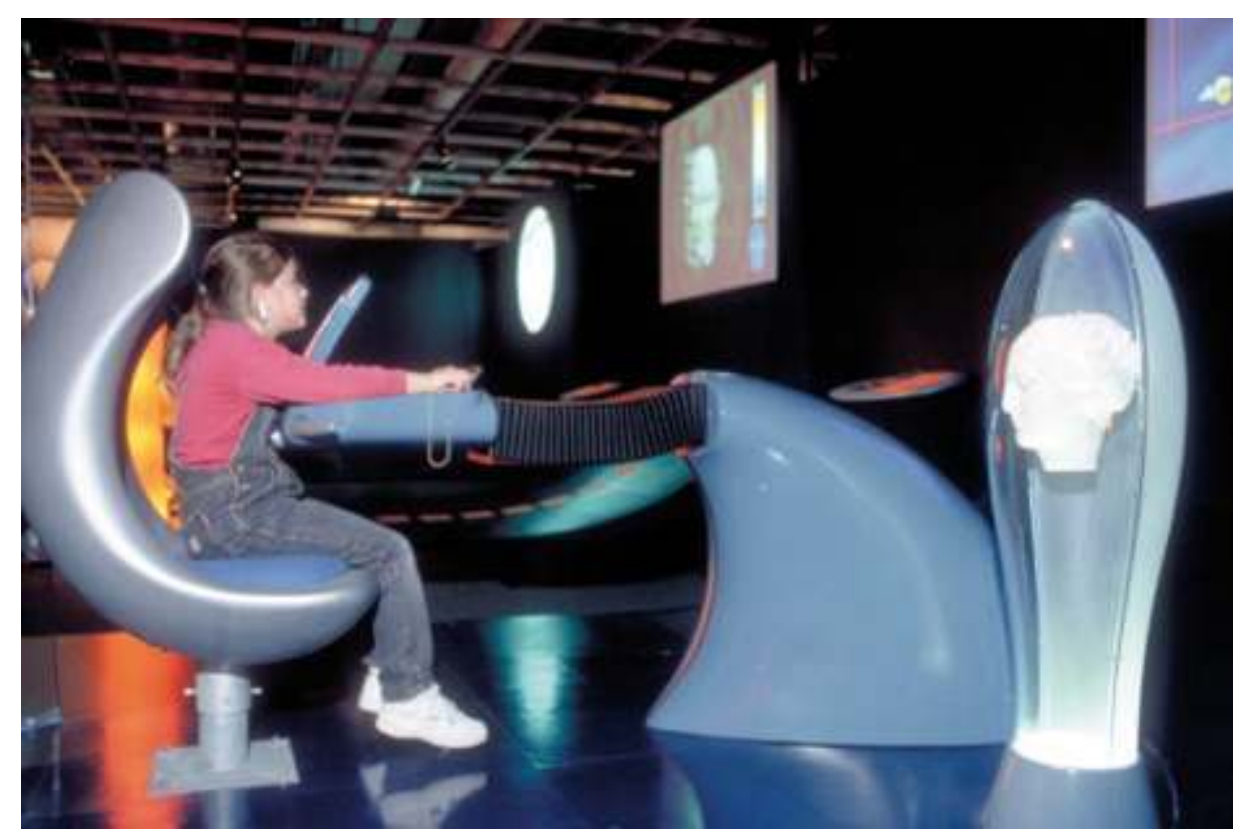

(c) EPPDCSI/Michel Lamoureux

\section{Troisième période : rationalisation, optimisation et diversification (2001 à 2016)}

Les quinze années qui suivent sont celles de la rationalisation, l'optimisation et la diversification des formes, styles et formats.

41 À partir de cette période, la tutelle budgétaire du ministère de la Culture s'avère prédominante même si le ministère de l'Enseignement supérieur et de la Recherche s'efforce de continuer à influer sur les grandes orientations de l'établissement.

42 L'accent est mis sur le développement de la fréquentation de la Cité des Sciences et de l'Industrie pour mieux remplir sa mission et hausser le niveau de ressources propres de l'établissement. Les analyses marketing sont plus développées et davantage prises en compte pour mieux répondre aux attentes des publics.

43 Ces évolutions du contexte conduisent à amplifier notre effort de rationalisation et d'optimisation de la programmation ainsi que de la production muséographique. De manière générale, qu'il s'agisse d'expositions permanentes ou temporaires :

45 - le public-cible est résolument enfants et familles, d'où une recherche de simplicité et d'immédiateté d'accès à l'offre ;

46 - les équipes muséographiques internes approfondissent leur professionnalisme et la muséographie vise davantage à l'efficacité communicationnelle ; moins d'éléments, plus fiables, plus grands, plus collectifs. 


\section{Les expositions permanentes $3^{\mathrm{e}}$ génération}

47 Malgré nos efforts, les publics continuent à réclamer un sens de visite plus clair. Il est alors proposé de réduire les surfaces des expositions permanentes pour mieux les circonscrire tandis qu'une nouvelle organisation des expositions permanentes sur 10000 $\mathrm{m} 2$ au lieu des $13000 \mathrm{~m}^{2}$ initiaux est mise en place suivant deux axes : "Des transports et des hommes". Ces renouvellements bénéficient d'un partenariat industriel très significatif. Mais il faut noter que, grâce aux approches science/société/ développement durable de chacun de ces sujets, de grands gestes scénographiques viennent remplacer les grands objets techniques des deux premières périodes d'Explora. Et c'était probablement une erreur car ces effets scénographiques n'ont pas pour le public la puissance d'attraction des grands objets. Heureusement, en 2014, le Solar Impulse 1, avec ses $60 \mathrm{~m}$ d'envergure, est installé sur la péri-trémie d'Explora.

51 En revanche, des trois grands récits projetés pour les expositions de référence, seul "Le grand récit de l'Univers" est créé. La forme du récit emprunte la modalité d'une enquête sur l'origine de la matière, dans un parcours à rebours du temps et de l'espace, du plus récent et plus proche au plus vieux et plus lointain. Le deuxième niveau de l'exposition témoigne d'une grande ambition de vulgarisation scientifique en tentant de rendre compréhensibles au grand public les grandes lois physiques de l'Univers au travers des 3 théories physiques: celle de Newton, la Relativité et la physique quantique.

"Le grand récit de la Vie" se concrétise par deux expositions distinctes :

- L'Homme et les gènes, développée en 2002 sous le commissariat scientifique d'Axel Kahn, entre dans la catégorie des expositions d'auteur. La muséographie est narrative et la scénographie l'accompagne avec un parcours linéaire très clair, en quatre salles, séparées par des sas de liaison récréatifs. Par ailleurs, un travail créatif est réalisé avec la cellule accessibilité de la Cité, notamment les éléments en résine créés pour les non-voyants, rendant également la manip plus lisible pour tous, comme le promeut le "design universel" que la Cité a fortement contribué à développer dans le secteur de la muséographie ;

54 - C3RV34U, l'expo neuro-ludique ouvre en 2014. Les scénographes de Scenorama se sont inspirés de l'univers des surréalistes pour évoquer la dimension imaginaire et créative du cerveau humain, évitant ainsi l'écueil d'une représentation trop mécaniste de ce sujet. L'exposition propose aux visiteurs des expériences et des tests directement issus de ceux pratiqués dans les laboratoires, accompagnés de commentaires par les chercheurs euxmêmes. 
"Le grand récit de l'Univers"

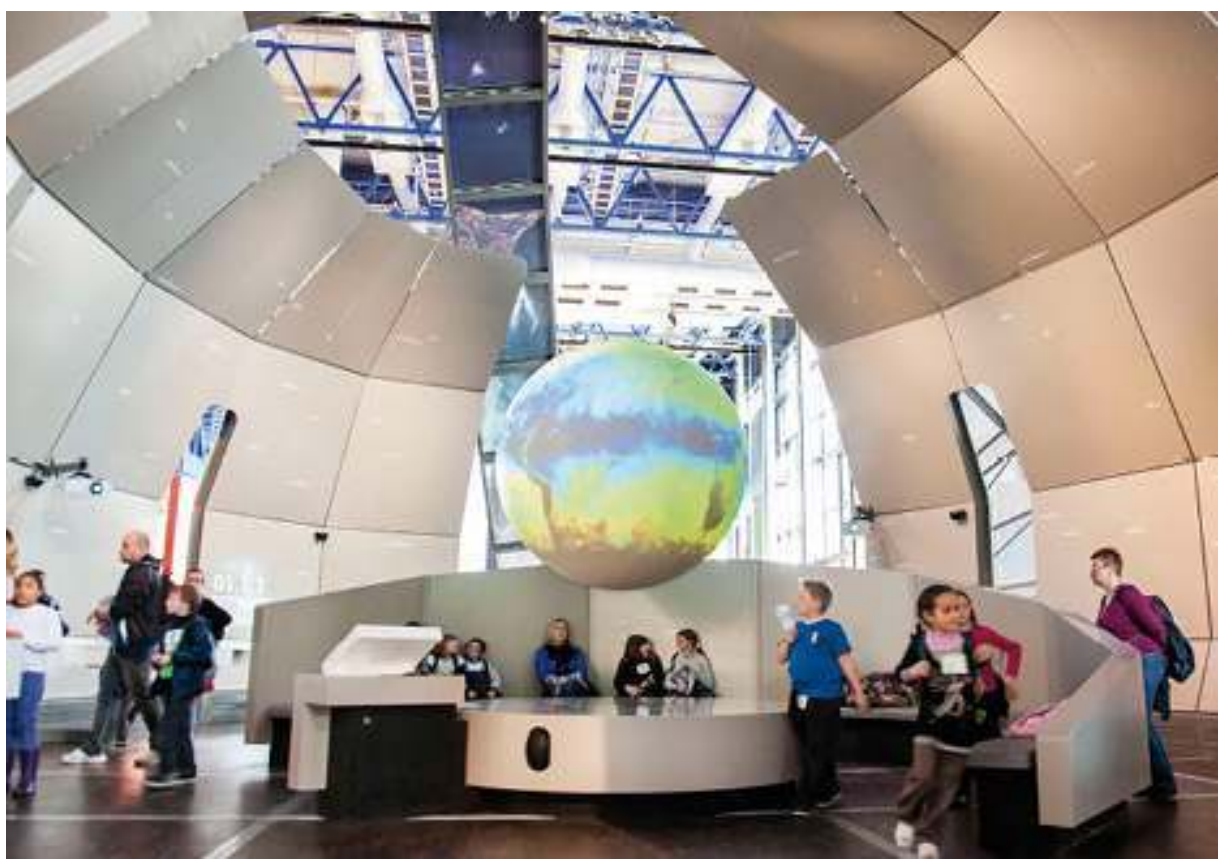

(c) EPPDCSI/Véronique Besnard

"Le grand récit de l'Univers"

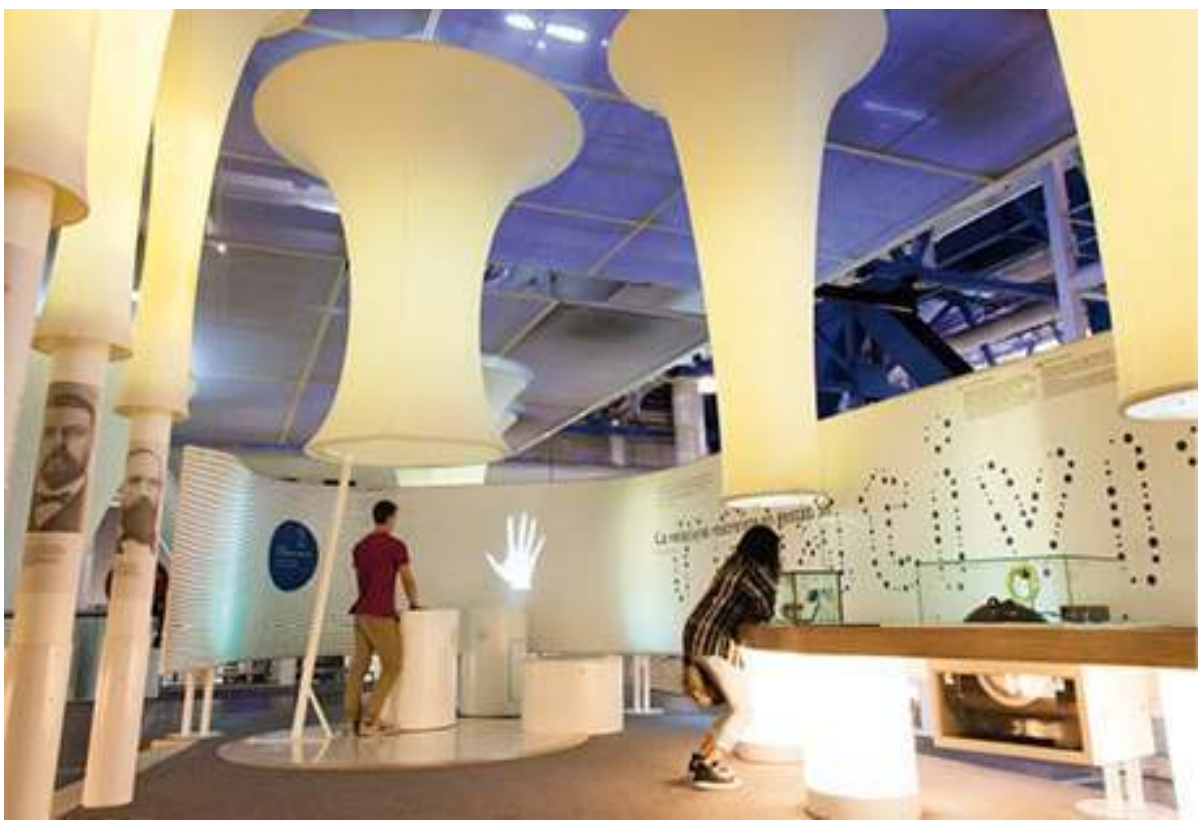

(c) EPPDCSI/Véronique Besnard

\section{Les expositions temporaires $3^{\mathrm{e}}$ période}

56 La politique d'expositions temporaires connaît un nouveau développement à partir de 2003, avec, on l'a vu, l'augmentation des surfaces allouées aux expositions temporaires aux dépens de celles allouées aux expositions permanentes. Une grille de programmation 
par saison de septembre à août est mise en place. Elle permet une programmation plus ajustée aux rythmes des visites. Elle prévoit deux grandes expositions par saison, la plus attractive en automne, l'autre de relance au printemps ; et en complément, des expositions d'opportunité itinérantes ou de partenariats sur des formats moyens.

Plusieurs stratégies de programmation sont mises en place.

\section{Une programmation adossée à de Grands Programmes de 2001 à 2006}

- "Les défis du vivant", avec en 2001 l'exposition L'Homme transformé dotée d'une scénographie expressionniste comprenant en son centre une installation interactive symbolisant une "peau" mi-biologique mi-numérique, réagissant aux déplacements des visiteurs. Par ailleurs, l'exposition présente des œuvres numériques interactives alors innovantes, comme l'installation Avolve utilisant des algorithmes de vie artificielle.

59 - "Gérer la Planète" avec en 2003, l'exposition Climax consacrée au réchauffement climatique servie, si l'on peut dire, par la crise caniculaire de 2003.

60 Une exposition dont les partis pris muséographiques radicaux sont dus à Winy Maas (agence MVRDV) : une exposition entièrement virtuelle, une échelle encore inédite dans l'immersion dans l'image (le grand film) et dans la projection des images de datavisualisation des simulateurs interactifs.

61 Ce programme se poursuit jusqu'en 2006 avec l'exposition Changer d'ère. Après Climax, qui avait été jugée "anxiogène" par une partie des visiteurs, cette exposition figure l'univers foisonnant des solutions possibles, dans une salle à la perspective augmentée par un paysage audiovisuel poétique occupant toute la longueur de la salle principale. Elle répond au désir des citoyens d'agir pour l'environnement à leur échelle.

62 Cette thématique est de nouveau reprise en 2011, avec Océan, climat \& nous. La salle d'introduction de l'exposition proposait un très grand dispositif immersif, à la dimension des enjeux écologiques liés au sujet et qui a eu un fort impact sur les visiteurs : en actionnant des capteurs au sol, ceux-ci pouvaient en s'approchant des écrans, ouvrir et refermer les eaux de l'Océan, pour révéler un écran illustrant les conséquences des activités humaines sur l'Océan.

63 Créée en 2016, l'exposition Mutations urbaines prolonge cette thématique essentielle du développement durable, avec notamment "Terre urbaine", un dispositif de datavisualisation et data-sonorisation projeté sur un écran hémisphérique de huit mètres de long, qui montre une réalité de la terre urbaine d'aujourd'hui au travers de la récolte de 130000 données à l'échelle planétaire. 


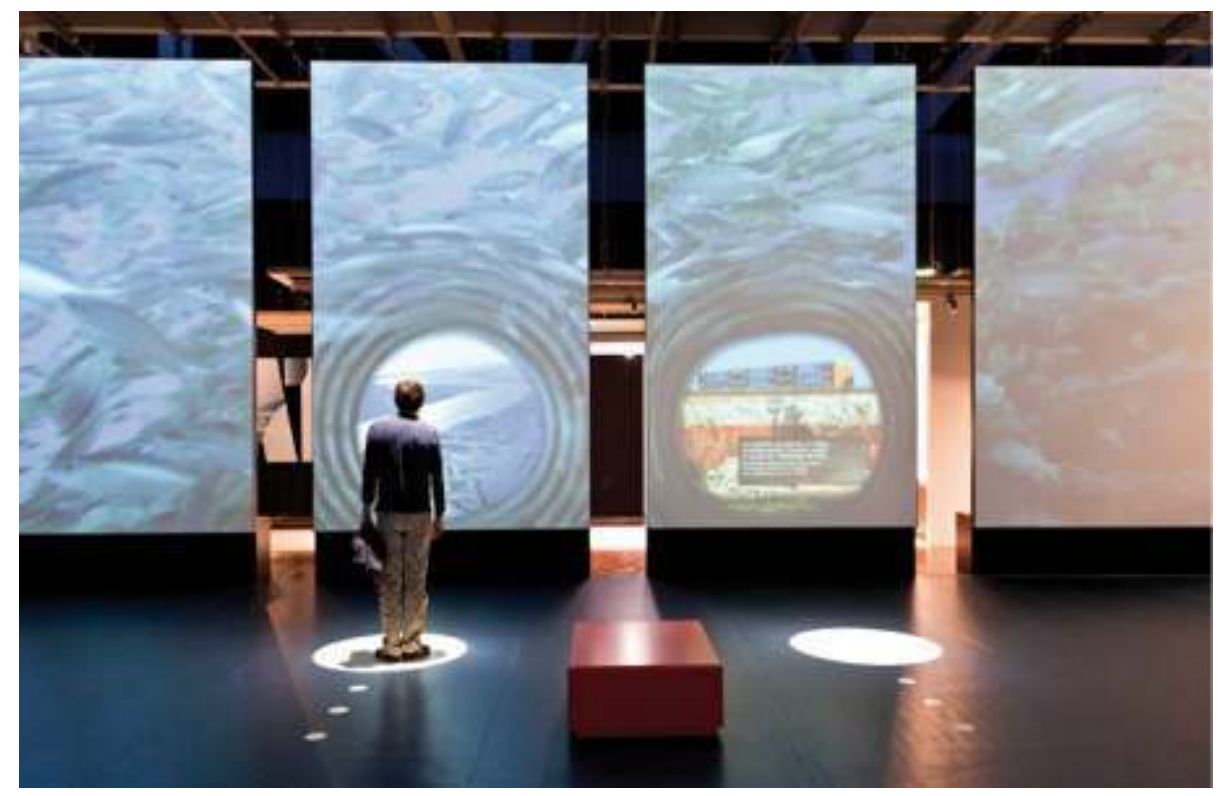

(c) EPPDCSI/Arnaud Robin

\section{La programmation opportuniste de "Blockbusters" et de "TGP" (expositions Très Grand Public)}

Il s'agit d'expositions adossées à des grands succès cinématographiques comme Trésors $d u$ Titanic en 2003 et surtout Star Wars, l'expo en 2005. Comment faire un blockbuster tout en restant fidèles à nos missions ? Pour une fois, nous pouvions nous appuyer sur l'attractivité d'une collection d'objets et nous avons développé un système d'interprétation très simple, même pas interactif, tirant deux fils : celui de l'histoire de 30 ans d'effets spéciaux et celui du regard du physicien Roland Lehoucq sur l'univers de Star Wars, permettant d'aborder de nombreuses notions scientifiques de manière ludique. Le point fort de cette exposition, une scénographie immersive à partir d'extraits des univers des différents épisodes de la saga. Une méga-exposition à la hauteur du mythe Star Wars et de la fréquentation escomptée. 


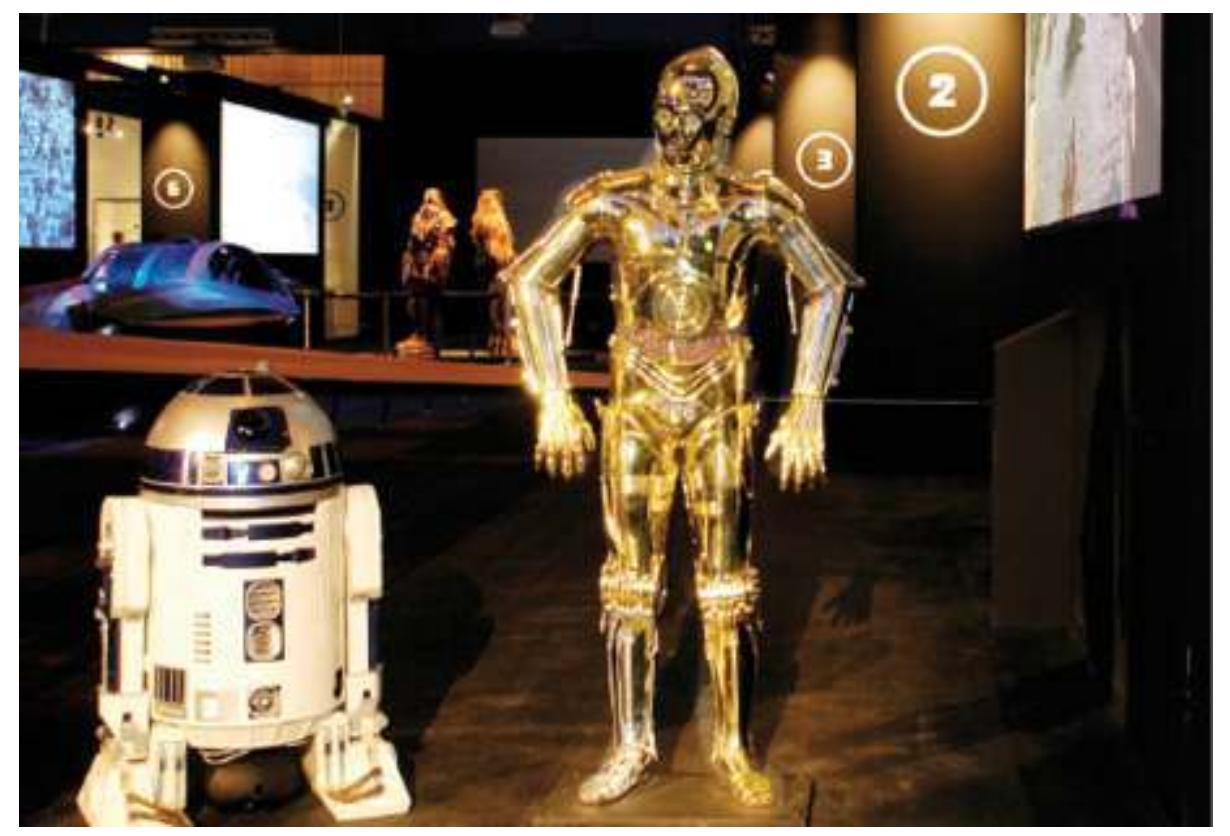

(c) EPPDCSI//Bernard Baudin

C'est aussi en 2010, Science \& Fiction, aventures croisées, une exposition née de la rencontre d'un passionné et de son extraordinaire collection mais aussi d'un besoin marketing : un bon sujet pour le grand public familial et une offre bonifiée par l'apport d'un partenariat fructueux au plan des œuvres présentées et des contenus avec la Bibliothèque nationale de France.

66 Mais c'est aussi des expositions dont l'attractivité était fondée sur l'innovation muséographique. Ce fut notamment le cas d'Epidemik, l'expo contagieuse, une exposition très innovante, entièrement virtuelle, avec un plateau de jeu interactif géant $\left(500 \mathrm{~m}^{2}\right)$ qui permettait au visiteur de devenir acteur au cœur d'une simulation de crise épidémique. En pénétrant sur le plateau, chaque visiteur découvrait au sol, attaché à ses pas, son "aura", un disque blanc porteur d'informations le concernant. Par ses déplacements, il faisait évoluer le scénario de crise au gré de ses différentes étapes. L'échelle de ce "serious game" était inédite, et n'a pas été reproduite jusqu'à ce jour.

Ce fut aussi une exposition basée sur une nouvelle pratique culturelle très populaire comme Jeu vidéo, l'expo, scénographie de l'agence Projectiles, inspirée de l'univers graphique des jeux vidéo dans un espace unique, cubique, entièrement tramé de lignes blanches lumineuses, faisant notamment référence à la grille de jeux du film Tron. Elle a ainsi proposé un espace de visite immersif et discursif, et non une simple juxtaposition de consoles de jeu. 


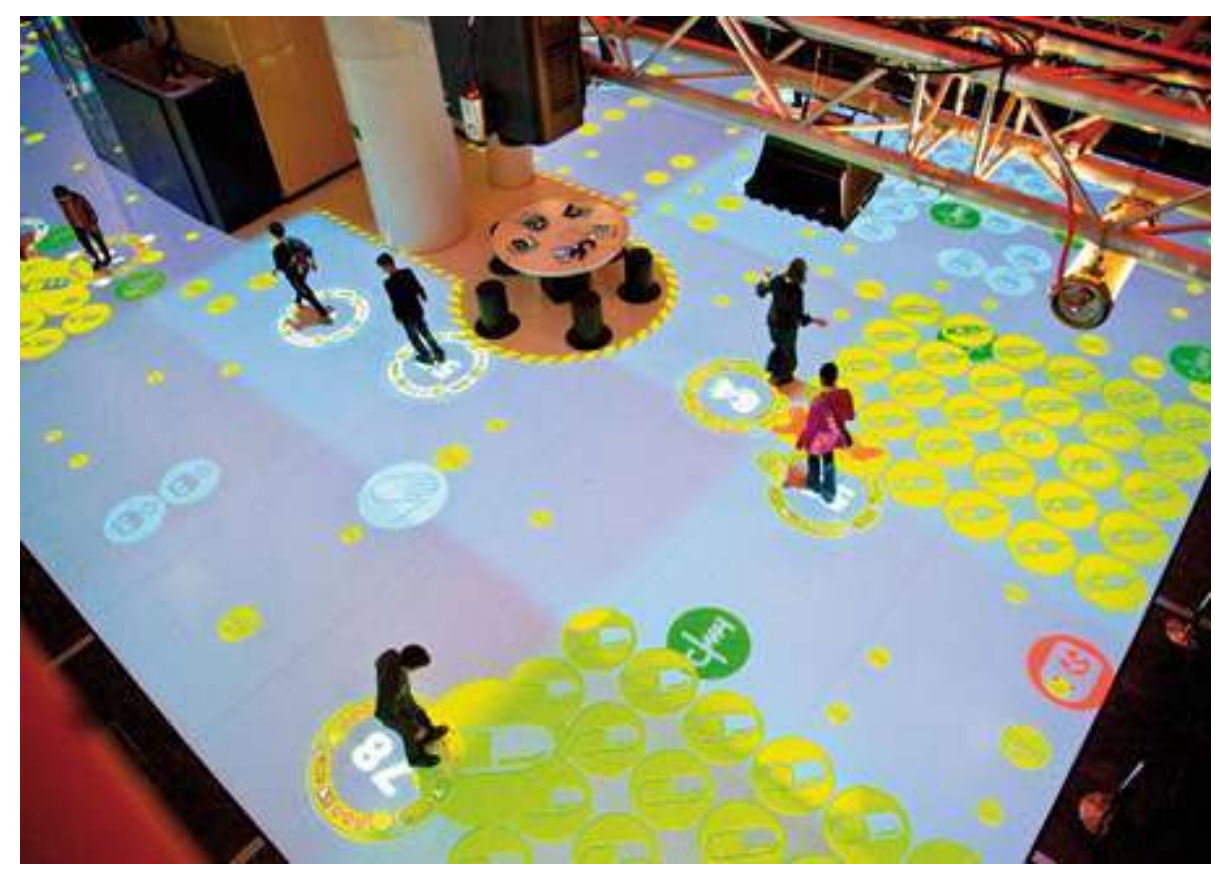

(C) EPPDCSI/S. Chiver

\section{Des "collections d'expos", à l'instar du monde de l'édition}

\section{Une collection d'Histoire culturelle des sciences}

Léonard de Vinci, projets, dessins, machines s'appuie sur une collection de maquettes historiques provenant du musée Leonard de Vinci de Milan et développe un système d'interprétation utilisant tous les ressorts de la muséographie interactive de la Cité. Une exposition qui mixe donc des procédés traditionnels de la muséographie de collection avec ceux de la muséographie interactive. Le tout dans une approche rigoureuse de la pensée et la démarche de Léonard de Vinci présentée dans son contexte historique. En prenant également la liberté d'un saut dans le monde contemporain et de la prolongation de la démarche d'observation de la nature de Léonard au travers des recherches actuelles en bio-mimétisme. Elle sera suivie de Darwin, l'original en 2016 et prochainement par une exposition consacrée à Louis Pasteur programmée en décembre 2017 au Palais de la découverte. Mais c'est aussi l'histoire revisitée par les sciences comme Gaulois, une expo renversante dans une scénographie de Pascal Payeur, suivie aujourd'hui par Quoi de neuf au Moyen-Âge?, toutes deux développées avec l'Inrap (Institut national de recherches archéologiques préventives).

\section{Une collection pour pré-ados/ados ou "familles orientées enfants"}

69 Elles créent un pont entre la Cité des Enfants et les autres offres en incitant les familles à visiter les autres expositions d'Explora. Elles sont adaptées à la muséographie interactive qui est la marque de fabrique de la Cité. Zizi sexuel, l'expo reprend les chapitres du Guide du Zizi Sexuel que Zep a conçu avec Hélène Bruller, pour parler de sexualité aux pré-ados, en répondant aux questions qu'ils se posent. Intéressant, le travail muséographique d'adaptation du livre: des manips surdimensionnées, des transpositions des dessins de 
Zep en éléments 3D interactifs mais aussi des créations totalement originales pour lesquelles Zep a fait de nouveaux dessins à la demande du commissariat. Une exposition qui a eu les honneurs du Los Angeles Times qui saluait "son courage, son caractère utilement éducatif et respectueux de la sensibilité des enfants". Suivie, un peu plus tard de Risque, osez l'expo, conçue par Universcience et coproduite avec les partenaires portugais (Ciencia Viva) et finlandais (Heureka) d'Universcience. Ce consortium de centres de science européens a ensuite coproduit Mental Désordre et Viral, l'expo contagieuse.

\section{Les expositions dites Art \& Sciences}

70 Citons Art Robotique en 2014, avec des œuvres monumentales qui portent sur leur transformation en autonomie comme le Totemobile de Chico Mac Murtrie, la matrix 3D de Christian Paros \& Shiro Takatani, l'Animaris de Théo Janssen, le Chemin de Damastes de Jean-Michel Bruyère.

\section{Vers la quatrième période...}

71 Un nouveau schéma directeur d'Explora prévoit une plus grande diversification des offres, une meilleure mise en valeur des espaces et des points de vues vers l'intérieur et l'extérieur et la reprise de l'installation de grands objets sur la galerie sud. Un programme de renouvellement progressif de la Cité des enfants est initié. Sous l'impulsion du président Bruno Maquart, les expositions temporaires sont programmées autour de six lignes éditoriales :

73 - "La science, toute une histoire !"/Grands acteurs et récits de science ;

74 - "Attention, science fraîche !" /Les dernières avancées de la recherche ;

75 - "Les mains dans le cambouis" /Le génie technique à l'œuvre ;

76 - "Sociétéscience"/Les mutations de notre monde ;

77 - "Chemins de traverse "/ La science là où on ne l'attend pas ;

- "Les petits curieux"/La science élémentaire pour les enfants.

79 En termes de muséographie, encore et toujours, il s'agit de renouveler et diversifier les formes, inventer, créer, innover! Comme le rappelle Michèle Antoine, directrice des expositions depuis juin 2016 :

"Les évolutions rapides des pratiques culturelles - et notamment numériques - de nos publics interrogent aujourd'hui l'exposition dans sa nature même de média. Nous devons réinventer cette forme en lui donnant une fonction d'espace public où dialoguent des approches différentes, où des points de vue s'entrechoquent. Nos propositions devront être hybrides et ouvertes afin que les publics s'en emparent de manière créative pour dialoguer entre eux, avec leurs pairs... et avec nous".

81 L'objectif initial de la Cité des Sciences et de l'Industrie, de rendre accessible à tous la culture scientifique et technique, reste un enjeu primordial. Malgré les politiques de programmation actives et diversifiées décrites tout au long de cet article, la fréquentation reste, comme pour les autres institutions culturelles, corrélée au niveau socio-culturel des visiteurs : les cadres et professions intellectuelles supérieures et les personnes diplômées sont venus trois fois plus que la moyenne des Français. Les enquêtes montrent 
également une fréquentation moins élevée pour les publics âgés de 15 à 30 ans et les personnes âgées de plus de 60 ans.

Dans la continuité du projet initial, mais dans un contexte socio-culturel qui s'est profondément transformé, il s'agira donc - comme l'indique le Projet d'établissement 2016-2020 Universcience - pour la prochaine décennie de mettre en œuvre, au travers d'une programmation inventive d'expositions mais aussi de toute une panoplie diversifiée d'autres offres, les valeurs de l'établissement : "ouverture, universalité, innovation".

Le texte suivant figure en encadré dans l'édition papier :

\section{Pour les enfants}

\section{La Cité des Enfants 1 ère génération en 1992}

La Cité des Enfants crée de toutes pièces une muséographie dédiée aux enfants et spécialement dessinée pour eux, esquissée dans l'Inventorium en 1986. Elle s'appuie sur les théories constructivistes de Jean Piaget et les concepts développés par André Giordan, dans une grande effervescence créative. Elle initie également une méthodologie de tests de prototypes par des enfants, avant la production de chaque élément d'exposition.

Des notions toujours valorisées aujourd'hui comme la coopération, l'action collective, sont mises en œuvre dans des éléments comme "Le Chantier" ou les "Jeux d'eau", dont le succès ne se dément pas avec de nombreuses duplications au plan national comme au plan international.

Des expositions temporaires pour enfants sont proposées en complément de la Cité des Enfants. Ainsi, Électricité, qu'y-a-t-il derrière la prise ? capte l'intérêt des enfants avec la création du petit personnage "Électro-toto" et innove en présentant une des premières manips hybridant réel et virtuel, où l'enfant, en tirant une corde réelle, fait se dérouler sur un écran toute la chaîne de distribution de l'électricité. Cette exposition connaîtra un gros succès à l'international avec trois duplications grâce au partenariat EDF.

\section{La Cité des Enfants $2^{\mathrm{e}}$ génération en 2007 et 2009}

87 Les surfaces sont doublées, les tranches d'âges allongées tandis qu'une structuration de l'espace beaucoup plus forte est apportée. Une allée centrale distribue en peigne les différents thèmes bien segmentés. On se démarque largement du vaste champ ouvert de manips que constituait la première Cité des Enfants. La scénographie cherche à s'éloigner des couleurs primaires habituellement réservées aux enfants. Elle fait des choix sobres pour les matériaux et les couleurs. Peu de texte, le graphisme compose avec des photographies d'enfants pour illustrer les thèmes et les modes d'emplois.

Après une interruption de huit ans, la programmation d'expositions temporaires enfants au rez-de-chaussée est reprise en 2004 et 2005. Ombres et Lumière, ouverte en 2005 et prolongée jusqu'en 2015, propose un parcours poétique et scientifique dans la maison du Professeur Ombre, dans un style encore inédit à la Cité, incluant des décors réalistes.

La programmation d'expositions temporaires enfants vient de nouveau d'être réactivée. 
Électricité, y a-t-il quelqu'un derrière la prise ?

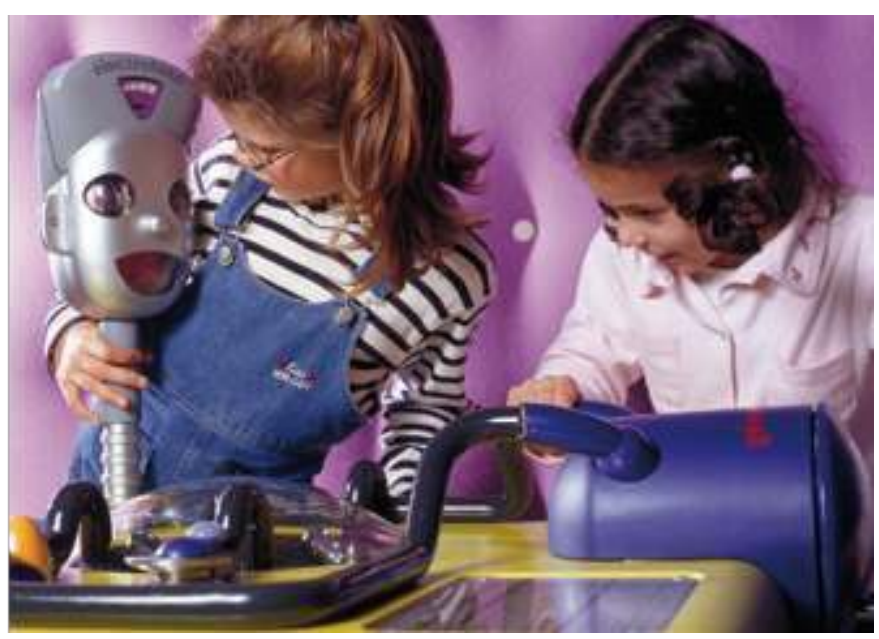

(c) EPPDCSI/Arnaud Baumann

Le texte suivant figure en encadré dans l'édition papier:

\section{Génériques des expositions citées}

La vigne et le vin, 1988 : Commissaire, Martine Thomas-Bourgneuf. Scénographe, Films et formes

Naissance d'un bateau, 1990 : Commissaire, François Vaysse. Scénographe, Mélusine

Machines à communiquer, 1991 : Commissaire, Marc Girard. Commissaire scientifique, Lucien Sfez. Commissaire artistique, Jean-Louis Boissier. Scénographe, Philippe Delis. Graphiste Integral, Ruedi Baur

Cité des enfants, 1992-1994 : Commissaires, Jean-Noël Plachez, Delphine Grinberg, Orna Cohen. Commissaire scientifique, Jacques Guichard. Scénographes, Xavier Leroux-Cauche, Oreste Friscira, Gilonne Brun

Emballe-moi ! 1994 : Commissaire, Françoise Thomas. Conception générale et scénographie, Caravanserail/Gérard Chollot, Tanguy Le Moing, François Jégou. Graphiste Visual Design, Jean Widmer

Mesures \& démesure, 1995 : Commissaire scientifique, Bernard Hagène. Chef de projet, Marie-Pierre Lahalle. Scénographe, Atelier Jean Nouvel, Hala Warde

Électricité, qu'y a-t-il derrière la prise ? 1996 : Commissaire, Orna Cohen. Scénographes, Pascal Payeur, Franck Fortecoeff. Designer, Michel Tortel

Nouvelles Images, Nouveaux Réseaux, 1997 : Commissaire, Marc Girard. Chefs de projet, Marie-Pierre Lahalle, Sabine Belloc, Virginio Gaudenzi. Scénographe, Reiner Werbitz. Concept salle de navigation, Maurice Benayoun. Graphiste, Michel Batory

Désir d'apprendre, 1999 : Commissaires, Orna Cohen, Marie-Pierre Lahalle, Blandine Savrda. Coordination, Françoise Thomas. Scénographes, Diane Chollet, Lucas Triboulet. Graphiste, Pierre Bernard

L'Homme transformé, 2001 : Commissaire, Françoise Thomas. Auteur, Joël de Rosnay. Scénographe, Zette Cazalas. Graphiste, Opixido 


\section{Auteurs
Solvit}

\section{Zizi sexuel, l'expo}

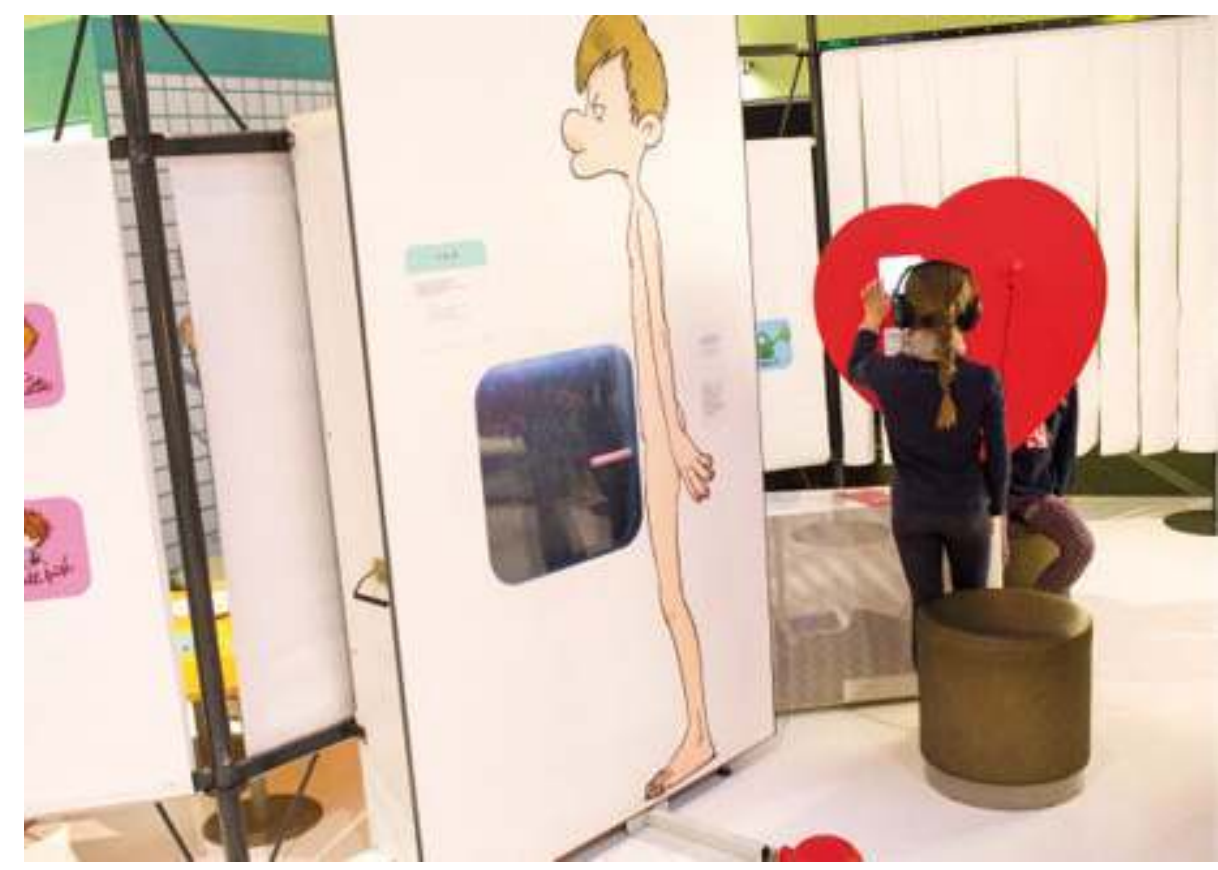

(c) EPPDCSI/Gilles Leimdorfer

L'Homme et les gènes, 2002 : Commissaire, Patrick Maury. Commissaire scientifique, Axel Kahn. Scénographe, Arnaud Sompairac. Graphiste, Gérard Plénacoste

Climax, 2003 : Commissaire, Marie-Pierre Lahalle. Expertise scientifique, Sylvestre Huet. Scénographe/Multimedia/Datavisualisation, MVRDV/Winy Maas

Ombres et lumière, 2005 : Commissaire, Xavier Limagne. Scénographe et graphiste, Lucie Lom

Star Wars, l'expo, 2005 : Commissaires, Sophie Lécuyer, Évelyne Hiard. Commissaire scientifique, Patrice Girod. Scénographes, Pylone Gaelle Seltzer, Jean-Pierre Boulanger

Changer d'ère, 2006 : Commissaire, Marie-Christine Hergault. Scénographe et graphiste, Projectiles. Design multimedia No design

Zizi sexuel, l'expo, 2007 : Commissaires, Maud Gouy, Dorothée Vatinel. Illustrateur et uteurs du guide, Zep et Hélène Bruller. Scénographe, Lucas Triboulet. Graphiste, Marion

Cité des enfants 2-7 ans, 2007 : Chef de projet, Marie-Pierre Lahalle. Commissaires, Orna Cohen, Sophie Bougé. Scénographe, Arnaud Sompairac. Designer, Agence Du\&Ma. Graphiste, Gérard Plénacoste

Le Grand Récit de l'Univers, 2008 : Commissaire, Patrick Maury. Commissaires scientifiques, Roland Lehoucq, Marc Lachière-Rey, Étienne Klein. Scénographes, Philippe Jeger, Philippe Merle. Graphiste, Gérard Plénacoste

Epidemik, 2008 : Commissaire, Anne Stéphan. Commissaires scientifiques, Antoine Flahaut et Patrick Zylberman. Scénographe, Stratosphère et Alain Domingo. Multimedia, Thierry Prieur, Olivier Feracci, Emmanuel Mââ Berriet. Graphiste, Jacques Boisnais 
110 Cité des enfants 5-12 ans, 2009 : Commissaire, Xavier Limagne. Scénographe, Nathalie Crinière. Designers, Johan Brunel, Samuel Misslen, Flavien Conilleau. Graphiste, Gérard Plénacoste

111 Océan, Climat \& Nous, 2011 : Commissaire, Marie-Christine Hergault. Scénographe, Arnaud Sompairac. Graphiste, Bénédicte Roland

112 Léonard de Vinci, projets, dessins, machines, 2012 : Commissaire, Éric Lapie. Commissaire scientifique, Claudio Giorgione. Scénographe, Franck Fortecoeff. Graphiste, Gérard Plénacoste

Léonard de Vinci, projets, dessins, machines

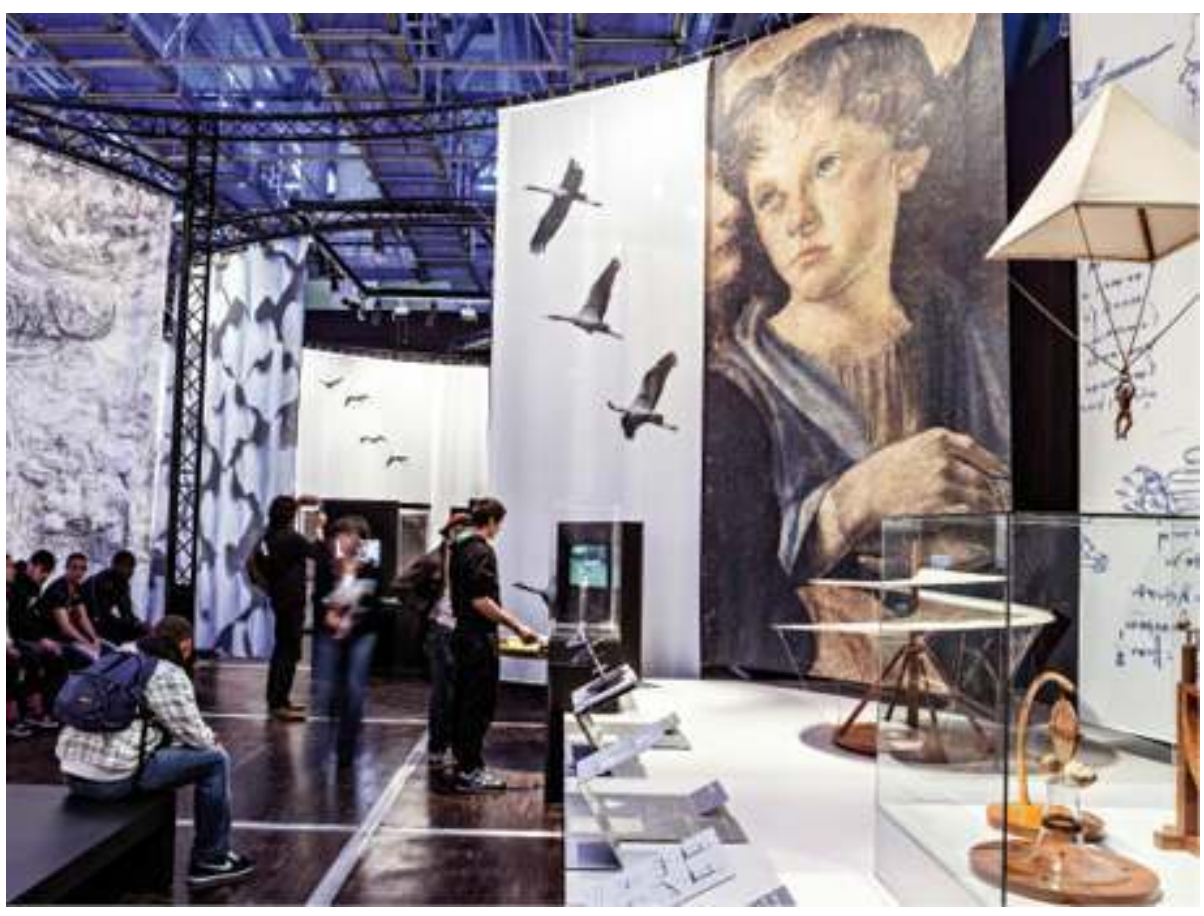

(c) EPPDCSI/Philippe Lévy

Jeu vidéo, l'expo, 2013 : Commissaire, Pierre Duconseille. Commissaires scientifiques, Mathieu Triclot, Olivier Lejade. Scénographe, Projectiles. Graphiste, Warmgrey/J.-J. Chapuis 


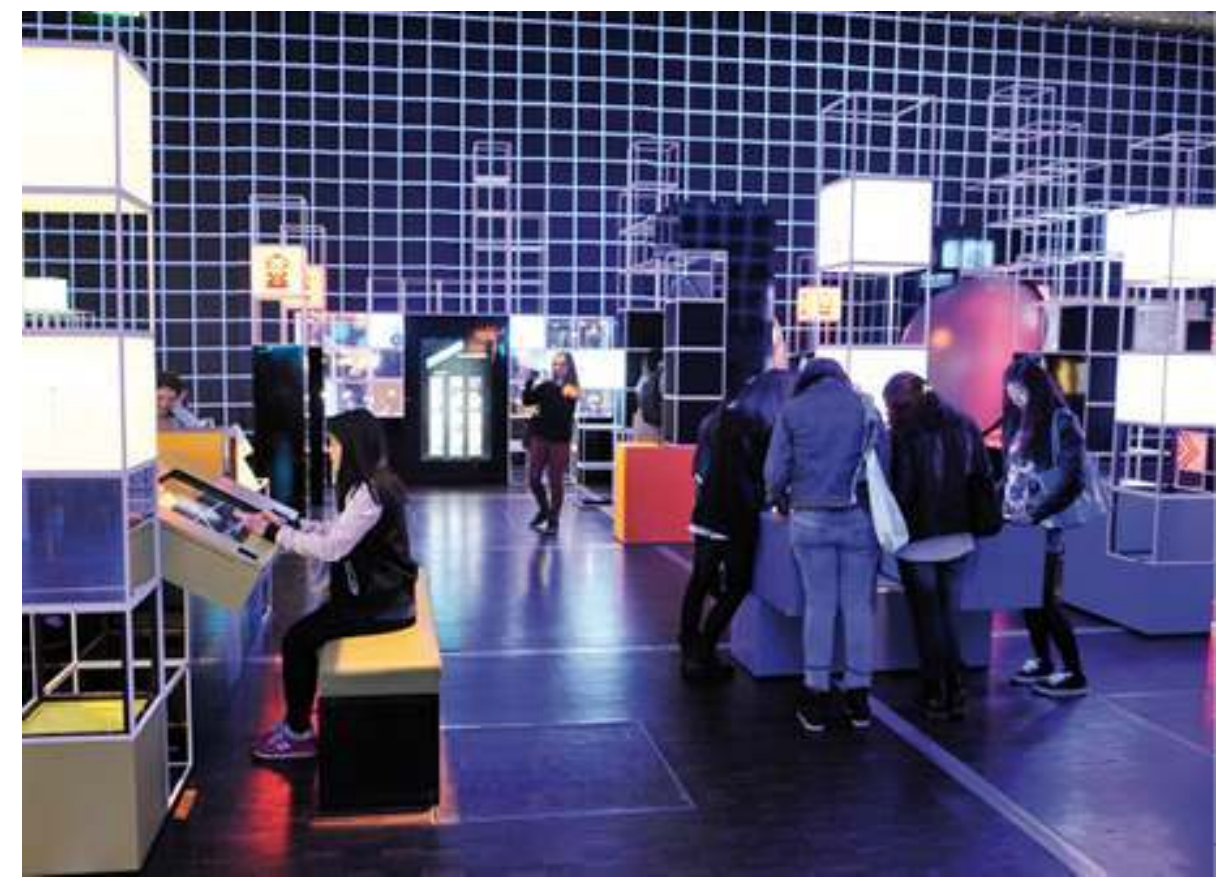

(c) EPPDCSI/Jean-Pierre Attal

114 C3RV34U, l'expo neuroludique, 2014 : Commissaire, Françoise Vallas. Commissaire scientifique, Stanislas Dehaene. Scénographe, Scenorama. Graphiste, Gérard Plénacoste

115 Art Robotique, 2014 : Commissaire artistique, Richard Castelli. Commissaire, Blandine Savrda. Scénographe, Lucas Triboulet. Graphiste, Catherine Martin

\section{Art robotique}

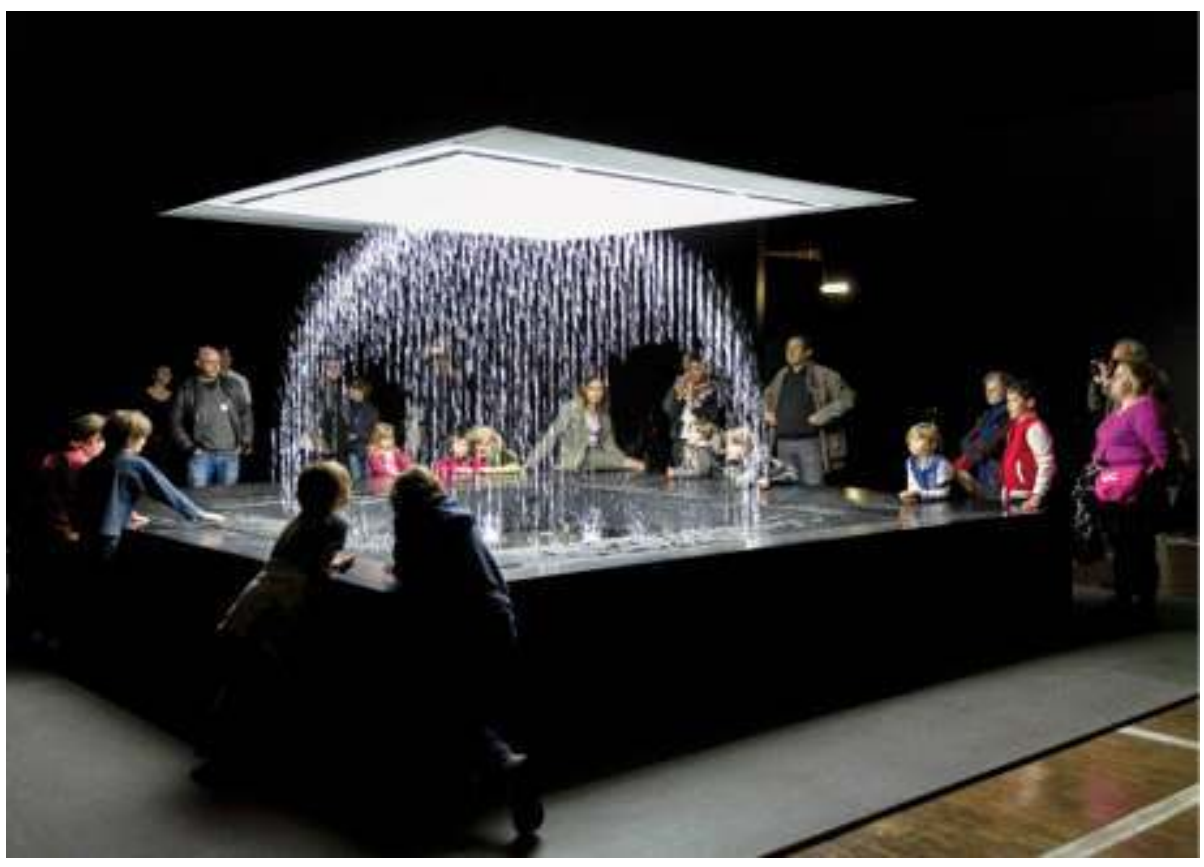

(C) EPPDCSI/Arnaud Robin 
116 Darwin, l'original, 2015 : Commissaire, Éric Lapie. Commissaire scientifique, Guillaume Lecointre. Scénographe, Projectiles. Graphiste, WA 75

117 Quoi de neuf au Moyen-Âge ? 2016 : Commissaires, Maud Gouy, Évelyne Hiard. Commissaire scientifique, Isabelle Catteddu. Scénographe, Paoletti-Rouland. Graphiste, Gérard Plénacoste

Quoi de neuf au Moyen-Âge?

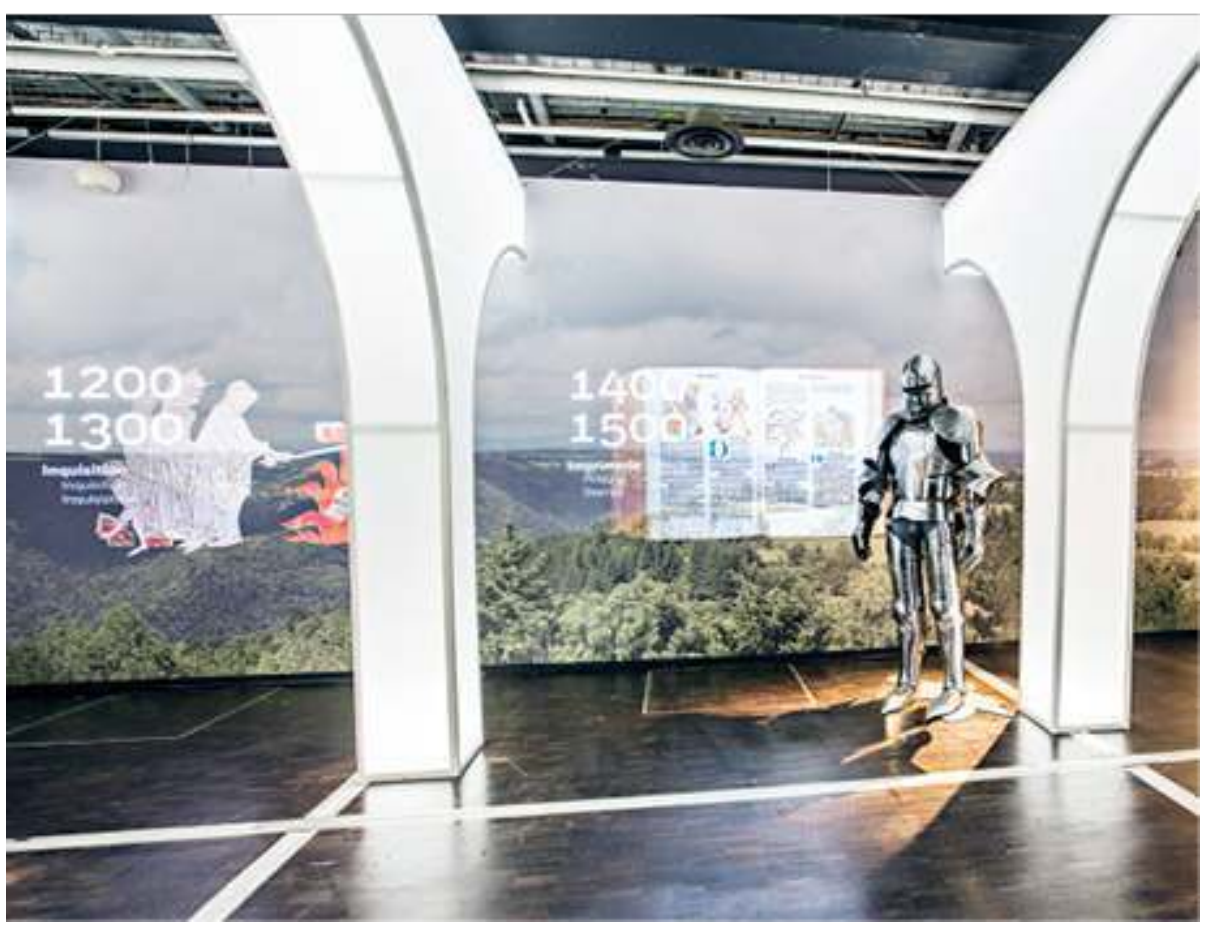

(c) EPPDCSI/Philippe Lévy

Mutations Urbaines, 2016 : Commissaire, Marie-Christine Hergault. Scénographes, AWP \& Nez Haut. Graphiste, Julie Linotte. Datavisualisation, Pascal Goblot

\section{INDEX}

Mots-clés: Exposition, muséographie, sciences et techniques

\section{AUTHORS}

\section{DOMINIQUE BOTBOL}

Chef du département des Projets muséologiques depuis 1998 et directrice adjointe des expositions depuis 2012

dominique.botbol@universcience.fr 
MARC GIRARD

Directeur des expositions de 1998 à 2015

DIANE CHOLLET

Architecte-scénographe, chef du département Production-Aménagements depuis 2014 